ISSN 2078-3744. Вісник Львів. ун-ту. Серія мех.-мат. 2018. Випуск 85. С. 60-65

Visnyk of the Lviv Univ. Series Mech. Math. 2018. Issue 85. P. 60-65

http://publications.lnu.edu.ua/bulletins/index.php/mmf

doi: http://dx.doi.org/10.30970/vmm.2018.85.060-065

УДК 517.5

\title{
A REMOVABILITY RESULT FOR SEPARATELY SUBHARMONIC FUNCTIONS
}

\author{
Juhani RIIHENTAUS \\ University of Eastern Finland, \\ P.O. Box 111, FI-80101, Joensuu, Finland, \\ and University of Oulu, \\ P.O. Box 3000, FI-90014, Oulun yliopisto, Finland, \\ e-mails: juhani.riihentaus@gmail.com, juhani.riihentaus@uef.fi
}

\begin{abstract}
Blanchet has shown that a $\mathcal{C}^{2}$ subharmonic function can be extended throught a $\mathcal{C}^{1}$ hypersurface provided the function satisfies certain $\mathcal{C}^{1}$ type continuity condtions on the exceptional hypersurface. Recently we improved Blanchet's result by measuring the exceptional set with the aid of Hausdorff measure. Now we give a related extension result for separately subharmonic functions.
\end{abstract}

Key words: subharmonic function, separately subharmonic function, Hausdorff measure, exceptional sets.

\section{INTRODUCTION}

1.1. We give an extension result for separately subharmonic $\mathcal{C}^{2}$ functions, see Theorem 2 below. Our proof is based on our previous extension result for $\mathcal{C}^{2}$ subharmonic functions, see [8, Theorem 1, p. 154], and on a general result, see [3, Proposition 1, p. 33]. Moreover, we need Federer's important results from the geometric measure theory, see e.g. [2, 9 .

1.2. For the used notation, see [6, 7, 8, However, for convenience of the reader we recall here the following: If $x=\left(x_{1}, \ldots, x_{n}\right) \in \mathbb{R}^{n}, n \geqslant 2$, and $j \in \mathbb{N}, 1 \leqslant j \leqslant n$, then we write $x=\left(x_{j}, X_{j}\right)=\left(X_{j}, x_{j}\right)$, where $X_{j}=\left(x_{1}, \ldots, x_{j-1}, x_{j+1}, \ldots, x_{n}\right)$. Moreover, if $A \subset \mathbb{R}^{n}$, $1 \leqslant j \leqslant n$, and $x_{j}^{0} \in \mathbb{R}, X_{j}^{0} \in \mathbb{R}^{n-1}$, we write

$$
A\left(x_{j}^{0}\right)=\left\{X_{j} \in \mathbb{R}^{n-1}: x=\left(x_{j}^{0}, X_{j}\right) \in A\right\}, \quad A\left(X_{j}^{0}\right)=\left\{x_{j} \in \mathbb{R}: x=\left(x_{j}, X_{j}^{0}\right) \in A\right\} .
$$

2010 Mathematics Subject Classification: 31B05, 31B25, 32A10, 32D20

(c) Riihentaus, J., 2018 


\section{Auxiliary Results}

2.1. A result of Federer. The following important result of Federer from the geometric measure theory will be used repeatedly.

Lemma 1 ([2, Theorem 2.10.25, p. 188], [9, Corollary 4, Lemma 2, p. 114]). Suppose that $E \subset \mathbb{R}^{n}, n \geqslant 2$. Let $\alpha \geqslant 0$ and let $\pi_{k}: \mathbb{R}^{n} \rightarrow \mathbb{R}^{k}$ denote the projection onto the first $k$ coordinates.

(i) If $\mathcal{H}^{k+\alpha}(E)=0$, then $\mathcal{H}^{\alpha}\left(E \cap \pi_{k}^{-1}(x)\right)=0$ for $\mathcal{H}^{k}$-almost all $x \in \mathbb{R}^{k}$.

(ii) If $\mathcal{H}^{k+\alpha}(E)<+\infty$, then $\mathcal{H}^{\alpha}\left(E \cap \pi_{k}^{-1}(x)\right)<+\infty$ for $\mathcal{H}^{k}$-almost all $x \in \mathbb{R}^{k}$.

2.2. Our previous extension result for subharmonic functions. As pointed out above, we use our previous extension result [8, Theorem 1, p. 154], however, now in the following, only seemingly more general form. For our previous related results, see [4, Theorem 4, pp. 181-182], [6, Theorem, p. 568], and [7, Lemma 2, p. 51]. Let it be pointed out also here that Blanchet's results [1, Theorems 3.1, 3.2 and 3.3, pp. 312-313], have been the starting point of our cited results.

Theorem 1. Suppose that $\Omega$ is a domain in $\mathbb{R}^{n}, n \geqslant 2$. Let $E \subset \Omega$ be closed in $\Omega$ and let $\mathcal{H}^{n-1}(E)<+\infty$. Let $u: \Omega \backslash E \rightarrow \mathbb{R}$ be subharmonic and such that the following conditions are satisfied:

(i) $u \in \mathcal{L}_{\text {loc }}^{1}(\Omega)$.

(ii) $u \in \mathcal{C}^{2}(\Omega \backslash E)$.

(iii) For each $j, 1 \leqslant j \leqslant n, \frac{\partial^{2} u}{\partial x_{j}^{2}} \in \mathcal{L}_{\text {loc }}^{1}(\Omega)$.

(iv) For each $j, 1 \leqslant j \leqslant n$, and for $\mathcal{H}^{n-1}$-almost all $X_{j} \in \mathbb{R}^{n-1}$ such that $E\left(X_{j}\right)$ is finite, the following condition holds:

For each $x_{j}^{0} \in E\left(X_{j}\right)$ there exist sequences $x_{j, l}^{0,1}, x_{j, l}^{0,2} \in(\Omega \backslash E)\left(X_{j}\right), l=1,2, \ldots$, such that $x_{j, l}^{0,1} \nearrow x_{j}^{0}, x_{j, l}^{0,2} \searrow x_{j}^{0}$ as $l \rightarrow+\infty$, and

$(i v(\mathrm{a})) \lim _{l \rightarrow+\infty} u\left(x_{j, l}^{0,1}, X_{j}\right)=\lim _{l \rightarrow+\infty} u\left(x_{j, l}^{0,2}, X_{j}\right) \in \mathbb{R}$

$(i v(\mathrm{~b}))-\infty<\liminf _{l \rightarrow+\infty} \frac{\partial u}{\partial x_{j}}\left(x_{j, l}^{0,1}, X_{j}\right) \leqslant \lim \sup _{l \rightarrow+\infty} \frac{\partial u}{\partial x_{j}}\left(x_{j, l}^{0,2}, X_{j}\right)<+\infty$.

Then $u$ has a subharmonic extension to $\Omega$.

2.3. In this connection and related to the above Theorem 1 , we take the opportunity to state the following concise corollary. As a matter of fact, we have previously not stated it explicitly, and we feel that it might be of interest in itself.

Corollary 1. Suppose that $\Omega$ is a domain in $\mathbb{R}^{n}, n \geqslant 2$. Let $E \subset \Omega$ be closed in $\Omega$ and let $\mathcal{H}^{n-1}(E)=0$. Let $u: \Omega \backslash E \rightarrow \mathbb{R}$ be subharmonic and such that the following conditions hold:

(i) $u \in \mathcal{L}_{\text {loc }}^{1}(\Omega)$

(ii) $u \in \mathcal{C}^{2}(\Omega \backslash E)$,

(iii) for each $j, 1 \leqslant j \leqslant n, \frac{\partial^{2} u}{\partial x_{j}^{2}} \in \mathcal{L}_{\text {loc }}^{1}(\Omega)$.

Then $u$ has a subharmonic extension to $\Omega$.

2.4. In addition to Federer's above lemma and our above Theorem 1, we need also the following nice result. Observe here that the below used hypoharmonic functions are in our terminology just subharmonic functions. 
Proposition 1 ([3, Proposition 1, p. 33]). Suppose that $\Omega$ is a domain in $\mathbb{R}^{p+q}, p, q \geqslant 2$. Let $w: \Omega \rightarrow[-\infty,+\infty)$ be nearly subharmonic. Let $w^{*}: \Omega \rightarrow[-\infty,+\infty)$ be the regularized function of $w$, which is then subharmonic. Then the following properties are equivalent.

(1) The distribution $\Delta_{x} w=\Delta_{x} w^{*}=$ (sum of the square second order derivatives of $w$ or $w^{*}$ with respect to the $p$ coordinates of $x$ ) is positive.

(2) For all $y \in \mathbb{R}^{q}$ the function $\Omega(y) \ni x \mapsto w^{*}(x, y) \in[-\infty,+\infty)$ is hypoharmonic.

(3) For almost every $y \in \mathbb{R}^{q}$ the function $\Omega(y) \ni x \mapsto w^{*}(x, y) \in[-\infty,+\infty)$ is subharmonic.

(4) For almost every $y \in \mathbb{R}^{q}$ the function $\Omega(y) \ni x \mapsto w^{*}(x, y) \in[-\infty,+\infty)$ is nearly subharmonic.

\section{AN EXTENSION RESULT FOR SEPARATELY SUBHARMONIC FUNCTIONS}

Our result is the following

Theorem 2. Suppose that $\Omega$ is a domain in $\mathbb{R}^{p+q}, p, q \geqslant 2$. Let $E \subset \Omega$ be closed in $\Omega$ and let $\mathcal{H}^{p+q-1}(E)<+\infty$. Let $w: \Omega \backslash E \rightarrow \mathbb{R}$ be separately subharmonic, that is,

for all $y \in \mathbb{R}^{q}$ the function $(\Omega \backslash E)(y) \ni x \mapsto w(x, y) \in \mathbb{R}$ is subharmonic,

and

for all $x \in \mathbb{R}^{p}$ the function $(\Omega \backslash E)(x) \ni y \mapsto w(x, y) \in \mathbb{R}$ is subharmonic, and such that the following conditions are satisfied:

(i) $w \in \mathcal{L}_{\text {loc }}^{1}(\Omega)$.

(ii) $w \in \mathcal{C}^{2}(\Omega \backslash E)$.

(iii) For each $j, 1 \leqslant j \leqslant p$, $\frac{\partial^{2} w}{\partial x_{j}^{2}} \in \mathcal{L}_{\mathrm{loc}}^{1}(\Omega)$, and for each $k, 1 \leqslant k \leqslant q$, $\frac{\partial^{2} w}{\partial y_{k}^{2}} \in \mathcal{L}_{\mathrm{loc}}^{1}(\Omega)$.

(iv) For each $j, 1 \leqslant j \leqslant p$, and for $\mathcal{H}^{p-1+q}$-almost all $\left(X_{j}, y\right) \in \mathbb{R}^{p-1+q}$ such that $E\left(X_{j}, y\right)$ is finite, the following condition holds:

For each $x_{j}^{0} \in E\left(X_{j}, y\right)$ there exist sequences $x_{j, l}^{0,1}, x_{j, l}^{0,2} \in(\Omega \backslash E)\left(X_{j}, y\right), l=$ $1,2, \ldots$, such that $x_{j, l}^{0,1} \nearrow x_{j}^{0}, x_{j, l}^{0,2} \searrow x_{j}^{0}$ as $l \rightarrow+\infty$, and

(iv(a)) $\lim _{l \rightarrow+\infty} w\left(x_{j, l}^{0,1}, X_{j}, y\right)=\lim _{l \rightarrow+\infty} w\left(x_{j, l}^{0,2}, X_{j}, y\right) \in \mathbb{R}$,

(iv(b)) $-\infty<\liminf _{l \rightarrow+\infty} \frac{\partial w}{\partial x_{j}}\left(x_{j, l}^{0,1}, X_{j}, y\right) \leqslant \lim \sup _{l \rightarrow+\infty} \frac{\partial w}{\partial x_{j}}\left(x_{j, l}^{0,2}, X_{j}, y\right)<+\infty$.

(v) For each $k, 1 \leqslant k \leqslant q$, and for $\mathcal{H}^{p+q-1}$-almost all $\left(x, Y_{k}\right) \in \mathbb{R}^{p+q-1}$ such that $E\left(x, Y_{k}\right)$ is finite, the following condition holds:

For each $y_{k}^{0} \in E\left(x, Y_{k}\right)$ there exist sequences $y_{k, l}^{0,1}, y_{k, l}^{0,2} \in(\Omega \backslash E)\left(x, Y_{k}\right), l=$ $1,2, \ldots$, such that $y_{k, l}^{0,1} \nearrow y_{k}^{0}, y_{k, l}^{0,2} \searrow y_{k}^{0}$ as $l \rightarrow+\infty$, and

$(v(\mathrm{a})) \lim _{l \rightarrow+\infty} w\left(x, y_{k, l}^{0,1}, Y_{k}\right)=\lim _{l \rightarrow+\infty} w\left(x, y_{k, l}^{0,2}, Y_{k}\right) \in \mathbb{R}$

$(v(\mathrm{~b}))-\infty<\lim \inf _{l \rightarrow+\infty} \frac{\partial w}{\partial y_{k}}\left(x, y_{k, l}^{0,1}, Y_{k}\right) \leqslant \lim \sup _{l \rightarrow+\infty} \frac{\partial w}{\partial y_{k}}\left(x, y_{k, l}^{0,2}, Y_{k}\right)<+\infty$.

Then $w$ has a separately subharmonic extension to $\Omega$.

Proof. By [5, Corollary 4.6, p. 412], $w$ is subharmonic in $\Omega \backslash E$. Thus by Theorem 1 $w: \Omega \backslash E \rightarrow \mathbb{R}$ has a subharmonic extension $w^{*}: \Omega \rightarrow[-\infty,+\infty)$. By Proposition 1 it is therefore sufficient to show that

- for $\mathcal{H}^{q}$-almost all $y \in \mathbb{R}^{q}$ the subharmonic function $(\Omega \backslash E)(y) \ni x \mapsto w(x, y) \in \mathbb{R}$ has a subharmonic extension $\Omega(y) \ni x \mapsto w^{*}(x, y) \in[-\infty,+\infty)$, and 
- for $\mathcal{H}^{p}$-almost all $x \in \mathbb{R}^{p}$ the subharmonic function $(\Omega \backslash E)(x) \ni y \mapsto w(x, y) \in \mathbb{R}$ has a subharmonic extension $\Omega(x) \ni y \mapsto w^{*}(x, y) \in[-\infty,+\infty)$.

We show that the first condition holds. The proof of the second is similar.

Fix $j, 1 \leqslant j \leqslant p$, arbitrarily for a while.

By our assumption $\mathcal{H}^{p-1+q}(E)<+\infty$. From the above Lemma of Federer, it follows that for $\mathcal{H}^{p-1+q}$-almost all $\left(X_{j}, y\right) \in \mathbb{R}^{p-1+q}$ the set $E(y)\left(X_{j}\right)$ is finite. Write

$$
A:=\left\{\left(X_{j}, y\right) \in \mathbb{R}^{p-1+q}: E(y)\left(X_{j}\right) \text { is finite }\right\} .
$$

Thus

$$
\mathcal{H}^{p-1+q}\left(A^{c}\right)=0 \Longleftrightarrow m_{p-1+q}\left(A^{c}\right)=0 \Longleftrightarrow \int_{\mathbb{R}^{p-1+q}} \chi_{A^{c}}\left(X_{j}, y\right) d m_{p-1+q}\left(X_{j}, y\right)=0,
$$

where $\chi_{A^{c}}(\cdot, \cdot)$ is the characteristic function of the set $A^{c}$, the complement taken in $\mathbb{R}^{p-1+q}$.

Next use Fubini's theorem:

$$
0=\int_{\mathbb{R}^{p-1+q}} \chi_{A^{c}}\left(X_{j}, y\right) d m_{p-1+q}\left(X_{j}, y\right)=\int_{\mathbb{R}^{q}}\left[\int_{\mathbb{R}^{p-1}} \chi_{A^{c}}\left(X_{j}, y\right) d m_{p-1}\left(X_{j}\right)\right] d m_{q}(y) .
$$

Since

$$
\int_{\mathbb{R}^{p-1}} \chi_{A^{c}}\left(X_{j}, y\right) d m_{p-1}\left(X_{j}\right) \geqslant 0
$$

we see that in fact

$$
\int_{\mathbb{R}^{p-1}} \chi_{A^{c}}\left(X_{j}, y\right) d m_{p-1}\left(X_{j}\right)=0
$$

for $\mathcal{H}^{q}$-almost all $y \in \mathbb{R}^{q}$.

Write

$$
\begin{aligned}
B_{1}^{j}: & =\left\{y \in \mathbb{R}^{q}: \int_{\mathbb{R}^{p-1}} \chi_{A^{c}}\left(X_{j}, y\right) d m_{p-1}\left(X_{j}\right)=0\right\}, \\
& =\left\{y \in \mathbb{R}^{q}: \chi_{A^{c}}\left(X_{j}, y\right)=0 \text { for } \mathcal{H}^{p-1} \text { almost all } X_{j} \in \mathbb{R}^{p-1}\right\}, \\
& =\left\{y \in \mathbb{R}^{q}: \chi_{A}\left(X_{j}, y\right)=1 \text { for } \mathcal{H}^{p-1} \text { almost all } X_{j} \in \mathbb{R}^{p-1}\right\} .
\end{aligned}
$$

Write $B_{1}:=B_{1}^{1} \cap B_{1}^{2} \cap \cdots \cap B_{1}^{p}$. Then for all $y \in B_{1}$ we have $\left(X_{j}, y\right) \in A$ for $\mathcal{H}^{p-1}$ almost all $X_{j} \in \mathbb{R}^{p-1}$, and this holds for all $j=1,2, \ldots, p$.

Next write

$$
\begin{aligned}
& B_{2}:=\left\{y \in \mathbb{R}^{q}: w(\cdot, y) \in \mathcal{L}_{\mathrm{loc}}^{1}(\Omega(y))\right\}, \\
& B_{3}:=\left\{y \in \mathbb{R}^{q}: w(\cdot, y) \in \mathcal{C}^{2}((\Omega \backslash E)(y))\right\}, \\
& B_{4}^{j}:=\left\{y \in \mathbb{R}^{q}: \frac{\partial^{2}}{\partial x_{j}^{2}} w(\cdot, y) \in \mathcal{L}_{\mathrm{loc}}^{1}(\Omega(y))\right\}, \\
& B_{4}:=B_{4}^{1} \cap B_{4}^{2} \cap \cdots \cap B_{4}^{p},
\end{aligned}
$$

and $B:=B_{1} \cap B_{2} \cap B_{3} \cap B_{4}$. 
Then for all $y \in B$ the function $(\Omega \backslash E)(y) \ni x \mapsto w(x, y) \in \mathbb{R}$ satisfies the assumptions of Theorem 1 . Therefore these functions have subharmonic extensions

$$
\Omega(y) \ni x \mapsto w^{*}(x, y) \in[-\infty,+\infty) .
$$

But then our claim follows from Proposition 1 .

Example 1. The function $u: \mathbb{R}^{4} \rightarrow \mathbb{R}$,

$$
u\left(z_{1}, z_{2}\right)=u\left(x_{1}+i y_{1}, x_{2}+i y_{2}\right)=u\left(x_{1}, y_{1}, x_{2}, y_{2}\right):= \begin{cases}1+x_{1}, & \text { when } x_{1}<0 \\ 1-x_{1}, & \text { when } x_{1} \geqslant 0\end{cases}
$$

is continuous in $\mathbb{R}^{4}$ and separately subharmonic, even separately harmonic in $\mathbb{R}^{4} \backslash(\{0\} \times$ $\mathbb{R}^{3}$ ), but not separately subharmonic in $\mathbb{R}^{4}$. Observe that $u$ satisfies the above conditions $(i),(i i),(i i i),(i v(\mathrm{a}))$ and $(v(\mathrm{a}))$ in $\mathbb{R}^{4} \backslash\left(\{0\} \times \mathbb{R}^{3}\right)$. However, $u \mid \mathbb{R}^{4} \backslash\left(\{0\} \times \mathbb{R}^{3}\right)$ does not satisfy the conditions $(i v(\mathrm{~b}))$ and $(v(\mathrm{~b}))$. Thus these conditions cannot be dropped in Theorem 2 .

Corollary 2. Suppose that $\Omega$ is a domain in $\mathbb{R}^{p+q}, p, q \geqslant 2$. Let $E \subset \Omega$ be closed in $\Omega$ and let $\mathcal{H}^{p+q-1}(E)=0$. Let $w: \Omega \backslash E \rightarrow \mathbb{R}$ be separately subharmonic, that is,

for all $y \in \mathbb{R}^{q}$ the function $(\Omega \backslash E)(y) \ni x \mapsto w(x, y) \in \mathbb{R}$ is subharmonic,

and

for all $x \in \mathbb{R}^{p}$ the function $(\Omega \backslash E)(x) \ni y \mapsto w(x, y) \in \mathbb{R}$ is subharmonic.

Suppose that the following conditions are satisfied:

(i) $w \in \mathcal{L}_{\text {loc }}^{1}(\Omega)$,

(ii) $w \in \mathcal{C}^{2}(\Omega \backslash E)$,

(iii) for each $j, 1 \leqslant j \leqslant p, \frac{\partial^{2} w}{\partial x_{j}^{2}} \in \mathcal{L}_{\text {loc }}^{1}(\Omega)$ and for each $k, 1 \leqslant k \leqslant q, \frac{\partial^{2} w}{\partial y_{k}^{2}} \in \mathcal{L}_{\text {loc }}^{1}(\Omega)$.

Then $w$ has a separately subharmonic extension to $\Omega$.

Proof. Follows directly from Theorem 2 and from the above Lemma of Federer.

\section{REFERENCES}

1. P. Blanchet, On removable singularities of subharmonic and plurisubharmonic functions, Complex Variables, Theory Appl. 26 (1995), no. 4, 311-322. DOI: 10.1080/17476939508814792

2. H. Federer, Geometric measure theory, Springer-Verlag, Berlin, 1969.

3. M. Hervé, Analytic and plurisubharmonic functions in finite and infinite dimensional spaces, Lect. Notes Math. 198. Springer, Berlin, 1971.

4. J. Riihentaus, Subharmonic functions, mean value inequality, boundary behavior, nonintegrability and exceptional sets, Workshop on Potential Theory and Free Boundary Flows; August 19-27, 2003, Kiev, Ukraine. In: Transactions of the Institute of Mathematics of the National Academy of Sciences of Ukraine, Kiev 1(2004), no. 3, 169-191.

5. J. Riihentaus, An inequality type condition for quasinearly subharmonic functions and applications, Positivity VII, Leiden, July 22-26, 2013, Zaanen Centennial Conference. In: Ordered Structures and Applications: Positivity VII, Trends in Mathematics, Springer Int. Publ., Berlin, 2016, pp. 395-414,

6. J. Riihentaus, Exceptional sets for subharmonic functions, Journal of Basic \& Applied Sciences 11 (2015), 567-571. DOI: 10.6000/1927-5129.2015.11.75 
7. J. Riihentaus, A removability result for holomorphic functions of several complex variables, Journal of Basic \& Applied Sciences 12 (2016), 50-52. DOI: 10.6000/1927-5129.2016.12.07

8. J. Riihentaus, Removability results for subharmonic functions, for harmonic functions and for holomorphic functions, Mat. Stud. 46 (2016), no. 2, 152-158. DOI: 10.15330/ms.46.2.152-158

9. B. Shiffman, On the removal of singularities of analytic sets, Michigan Math. J. 15 (1968) no. $1,111-120$. DOI: $10.1307 / \mathrm{mmj} / 1028999912$

Стаття: надійшла до редколегії 04.07.2018

доопращъована 27.09.2018

прийнята до друку 26.12.2018

\section{ОДИН РЕЗУЛЬТАТ ПРО УСУНЕННЯ ОСОБЛИВОСТЕЙ ДЛЯ НАРІЗНО СУБГАРМОНІЧНИХ ФУНКЦІЙ}

\section{Юхані РІІГЕНТАУЗ}

University of Eastern Finland, P.O. Box 111, FI-80101, Joensuu, Finland, and University of Oulu, P.O. Box 3000, FI-90014, Oulun yliopisto, Finland, e-mail: juhani.riihentaus@gmail.com, juhani.riihentaus@uef.fi

Бланше довів, що субгармонічну функцію гладкості $\mathcal{C}^{2}$ можна продовжити через $\mathcal{C}^{1}$-гіперплощину, якщо функція задовольнє певну умову гладкості типу $\mathcal{C}^{1}$ на винятковій гіперплощині. Нещодавно ми покращили результат Бланше, розглянувши міру Гаусдорфа виняткової множини. Тепер ми наводимо подібне узагальнення для нарізно субгармонічних функцій.

Ключові слова: субгармонічна функція, нарізно субгармонічна функція, міра Гаусдорфа, виняткові множини. 